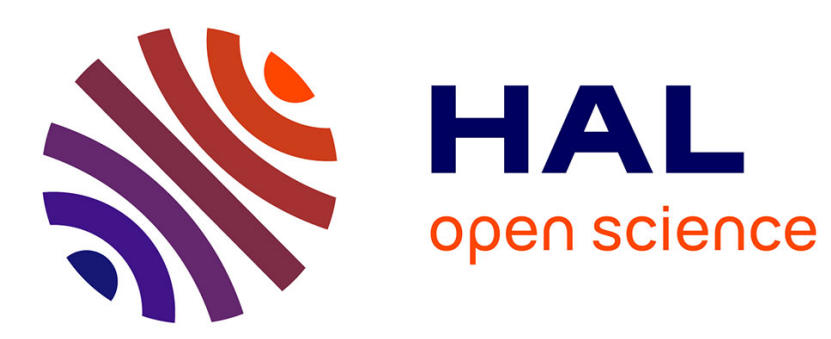

\title{
Fractional Bit Encoded Spatial Modulation (FBE-SM)
}

Nikola Serafimovski, Marco Di Renzo, Sinan Sinanovic, Raed Mesleh, Harald

Haas

\section{To cite this version:}

Nikola Serafimovski, Marco Di Renzo, Sinan Sinanovic, Raed Mesleh, Harald Haas. Fractional Bit Encoded Spatial Modulation (FBE-SM). IEEE Communications Letters, 2010, 14 (5), pp. 429-431. hal-00547012

\section{HAL Id: hal-00547012 \\ https://hal.science/hal-00547012}

Submitted on 15 Dec 2010

HAL is a multi-disciplinary open access archive for the deposit and dissemination of scientific research documents, whether they are published or not. The documents may come from teaching and research institutions in France or abroad, or from public or private research centers.
L'archive ouverte pluridisciplinaire HAL, est destinée au dépôt et à la diffusion de documents scientifiques de niveau recherche, publiés ou non, émanant des établissements d'enseignement et de recherche français ou étrangers, des laboratoires publics ou privés. 


\title{
Fractional Bit Encoded Spatial Modulation (FBE-SM)
}

\author{
N. Serafimovski, M. Di Renzo, S. Sinanović, R. Y. Mesleh, and H. Haas
}

\begin{abstract}
In this Letter, we introduce fractional bit encoded (FBE)-spatial modulation (SM), which is a novel and more versatile SM scheme that allows the transmitter to be equipped with an arbitrary number of antennas. The solution is based on the theory of modulus conversion, and is especially useful for compact mobile devices where cost and space constraints pose fundamental limits on the achievable bit rate. Numerical results will show that FBE-SM can offer design flexibility and the desired trade-off in terms of attainable performance and capacity.
\end{abstract}

Index Terms-Spatial modulation (SM), Multiple-inputmultiple-output (MIMO) systems, modulus conversion.

\section{INTRODUCTION}

$\mathbf{T}$ HE aim of this Letter is to propose a method for overcoming the limitation on the number of transmit antennas in SM and allow the transmitter to be equipped with an arbitrary number of antennas. SM is a novel approach to multiple-input-multiple-output (MIMO) systems which entirely avoids inter-channel interference (ICI) and requires no synchronisation between the transmit antennas, while achieving a spatial multiplexing gain. This is performed by mapping a block of information bits into a constellation point in the signal and spatial domains [1]. In SM, the number $k$ of information bits that are encoded in the spatial domain is directly related to the number $M$ of transmit antennas, in particular $M=2^{k}$. This means that the number of transmit antennas must be a power of two. We propose a solution to this limitation in SM which increases the granularity of the data encoding process in the spatial domain by using fractional bit encoding; the novel method is called FBE-SM.

When applied to SM, FBE relies on encoding each point in the spatial domain, i.e., the antenna index, with, on average, a non-integer number of bits, while keeping unchanged the encoding process in the signal domain. This results in a more versatile system design allowing for a wider range of spectral efficiencies given restrictions on space and power consumption [1]. By using FBE-SM, a system can achieve otherwise unavailable data rates in the spatial domain. For example, it may not be possible to install 8 antennas in a small-scale portable device, while 5 or 6 might be feasible. In such a case, however, SM would use only 4 antennas [1]. FBE-SM is designed to address this exact problem.

The concept behind FBE is not new in modern communication systems. However, to the best of the authors' knowledge,

Manuscript received November 19, 2009. The associate editor coordinating the review of this letter and approving it for publication was J. Jalden.

N. Serafimovski, S. Sinanović, and H. Haas are with The University of Edinburgh, Edinburgh, EH9 3JL, UK (e-mail: \{n.serafimovski, s.sinanovic, h.haas\}@ed.ac.uk.).

M. Di Renzo is with L2S, UMR 8506 CNRS - SUPELEC - Univ ParisSud, 3 rue Joliot-Curie, 91192 Gif-sur-Yvette CEDEX, France (e-mail: marco.direnzo@1ss.supelec.fr).

R. Y. Mesleh is with Jacobs University Bremen, 28759 Bremen, Germany (e-mail: r.mesleh@jacobs-university.de).

Digital Object Identifier 10.1109/LCOMM.2010.05.092270 this principle has never been applied to SM. For example, the application of FBE to a pulse amplitude modulation (PAM) communication system is reported in [5]. Two general methods for fractional bit transmission are described therein. The first approach is called constellation switching which alternates between the transmission of $B$ and $B+1$ bits per symbol to achieve the FBE over time. A notable downside of this approach is the inherent bit shift that results from incorrectly decoded symbols making it prone to error propagation effects. The second approach is called modulus conversion and is designed to minimise the error propagation effect that afflicts the performance of the constellation switching method [6]. In this Letter, we apply the theory of modulus conversion to SM and propose a system that can offer satisfactory performance for an arbitrary number of antennas at the transmitter.

The remainder of this Letter is organized as follows. In Section II, the theory of modulus conversion is briefly summarised. In Section III, the novel FBE-SM scheme is introduced. In Section IV, some numerical results are shown to analyse the performance of FBE-SM and compare it with conventional SM. Finally, Section V concludes the Letter.

\section{THEORY OF MOdulus CONVERSION}

Modulus conversion achieves fractional bit rates by converting the incoming bitstream to numbers in an arithmetic base, or modulus, that is not a power of 2 [6]. In particular, the modulus converter operates as follows: i) blocks of $S K$ bits are extracted from the incoming bitstream, where $K$ is the desired fractional bit rate and $S$ is a positive integer; ii) each block is then converted to $S$ numbers of base $L$. The modulus is defined as the smallest integer number, $L$, such that $L \geq 2^{K}$.

In general, one may use the theory of modulus conversion to achieve an arbitrary fractional bit rate, i.e., $K$ could be a real number. In Section III, we will see that this is especially relevant when applying this method to SM. However, since both $L$ and $S$ are positive integer numbers it follows that the modulus converter can only handle rational bit rates, i.e., $K \in \mathbb{Q}$. This problem can be circumvented by approximating any $K$, with $\tilde{K}$, given as the ratio of two positive and relatively prime integers $p$ and $q$. In other words, the problem can be stated as finding the best rational approximation, $\tilde{K}=p / q$, to a real number $K$. A simple way to do that is to exploit the following inequality:

$$
0 \leq n K-\lfloor n K\rfloor \leq 1 \Rightarrow 0 \leq K-\frac{\lfloor n K\rfloor}{n} \leq \frac{1}{n}
$$

where $\lfloor\cdot\rfloor$ denotes the floor function and $n$ is an arbitrary and positive integer number.

From (1), it follows that $S K \cong S \tilde{K}=S(\lfloor n K\rfloor / n)$, which, according to the theory of modulus conversion, must be a positive integer. It is worth mentioning that, in general, $S \neq n$. In particular, although $n$ and $S$ could be arbitrarily chosen, 
the following guidelines can be considered for their optimal setting:

1) The larger $n$ is, the smaller the approximation error $K-\tilde{K}$ is; this follows from (1).

2) The larger $S$ is, the longer the decoding delay is: this follows from the operating principle of modulus conversion, which allows the receiver to decode the data only after receiving $S$ base- $L$ numbers.

3) The larger $S$ is, the greater the vulnerability to error propagation within each block of bits (see Section IV).

Accordingly, for any given $K$, and provided that $S(\lfloor n K\rfloor / n)$ is a positive integer, $n$ and $S$ should be chosen as large and as small as possible, respectively.

\section{Application of Modulus Conversion to SM}

Let us now apply the theory of modulus conversion to SM and introduce the FBE-SM method. As mentioned in Section I, the motivation for the proposed scheme is to avoid fundamental constraints on the number of transmit antennas that can be used by classical SM systems. We emphasize that the proposed method applies to only the bit encoding in the spatial domain, while the encoding process in the signal domain is left unchanged. FBE-SM reduces to conventional SM [1] if the number of antennas is a power of 2 .

\section{A. FBE-SM: A Step-by-Step Description}

The working principle of FBE-SM can be summarised in the following subsequent steps:

1) Determine the desired number of transmit antennas, $M$, according to the system constraints, e.g., bit rate, cost, available space.

2) Set the modulus $L$ in Section II equal to $M$, i.e., $L=M$.

3) Compute the maximum spatial multiplexing gain offered by the system as $K=\log _{2}(M)$.

4) Choose the pair $(S, n)$ such that $S(\lfloor n K\rfloor / n)$ is a positive integer number and following the design guidelines described in Section II, i.e.:

- Optimize $\tilde{K}=\lfloor n K\rfloor / n$ such that it is as close as possible to $K$. This allows the system to approach the spatial multiplexing gain offered by the $M$ transmit antennas. This is achieved, in general, for larger values of $n$.

- Optimize $S$ such that it is as small as possible: this reduces the decoding delay and, more importantly, minimises error propagation in the decoded bitstream.

5) Map each of the $S$ base- $M$ encoded numbers in the transmission block to a transmit antenna index in the range $[0, M-1]$.

The receiver will perform the reverse operation to recover the encoded data:

i) for each signaling interval, one of the algorithms in [1] [3] is applied to detect the spatial and signal constellation points, ii) the spatial constellation points (the base- $M$ encoded numbers) are grouped into blocks of $S$ points each, and iii) each block is converted to the equivalent base-2 bitstream of $S(\lfloor n K\rfloor / n)$ bits each. At this point it should be noted that these are merely guidelines to achieve a balance between the best approximation of $K$ and minimising the error propagation effects. The best rational approximation for any real number is obtained using continuous fractions [7] given the restriction on the available block size $n$.

\section{B. FBE-SM: An Example}

Let us consider a simple example with $M=5$ without signal modulation: this is equivalent to considering the space shift keying (SSK) modulation method [3]. Thus, we have $K=2.3219$. By choosing, e.g., $(S, n)=(4,4)$, we get $\tilde{K}=2.25$, which closely approaches $K$ and is greater than the spatial multiplexing gain offered by a system with $M=4$. If, for instance, the block of $S \tilde{K}$ bits is equal to $110001011_{2}$, then the modulus converter will return an $(S \tilde{K})_{M}$ block equal to $3040_{5}$, where $(x)_{b}$ denotes the base- $b$ representation of $x$. Then, the output of the modulus converter is mapped to a spatial constellation point. First, the antenna with index 3 transmits an energy signal, then the antenna with index 0 transmits the same signal, etc. The receiver will estimate each received antenna index by using an index-by-index detection algorithm according to [1]-[3]. After decoding the $S$ antenna indexes, ideally with no errors, it will recover the original data stream as: $3040_{5}=110001011_{2}$.

In order to understand the effect of error propagation, let us consider that an error has occurred in the detection of the second antenna index. Let us assume that the decoded block is $3140_{5}$. In this case, the decoded bitstream will be $3140_{5}=110100100_{2}$, which will result in 5 out of 9 bits in error. In particular, the error propagation effect in the last 4 digits is well evident in this case. This example highlights the important role played by $S$ to limit the error propagation effect in the decoded bitstream for each incorrectly decoded base$M$ number in the received block. However, numerical results in Section IV will show that for moderately high signal-tonoise-ratios (SNRs) the performance degradation due to error propagation is not significant.

\section{NUMERICAL EXAMPLES}

The following system setup is considered: i) Each transmit antenna, when activated, transmits a 4-QAM (quadrature amplitude modulation) signal. ii) The channel is assumed to be Rayleigh distributed with uncorrelated fading among the wireless links. It is static and flat-fading for the duration of a transmission block. iii) The noise at the receiver input is assumed to be white complex Gaussian, with zero-mean and mutually independent samples. iv) The receiver is equipped with 4 antennas and uses a maximum-likelihood detector to jointly detecting spatial and signal constellation points [2].

Two performance metrics will be investigated: 1) the symbol-error-ratio (SER), which is defined as the average probability of incorrectly detecting a constellation and signal point [1], and 2) the bit-error-ratio (BER), which is defined as the average probability of incorrectly detecting a bit in the decoded bitstream [2]. This allows us to better highlight the effect of error propagation introduced by the FBE process.

In Fig. 1, we analyse the BER of FBE-SM for various combinations of $(S, n)$ and $M=5$ in order to substantiate the claims in Section II and Section III. We observe that the BER gets progressively worse for increasing values of $S$ due 


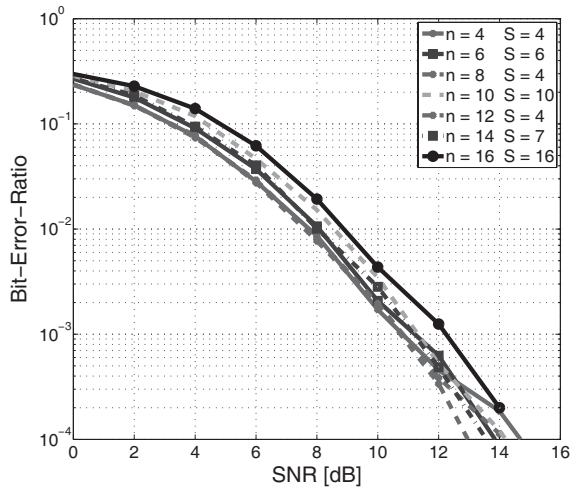

Fig. 1. BER of FBE-SM. Parametric study for different values of $(S, n)$. Setup: i) $M=5$, and ii) $K=2.3219 \mathrm{bits} / \mathrm{s} / \mathrm{Hz}$.

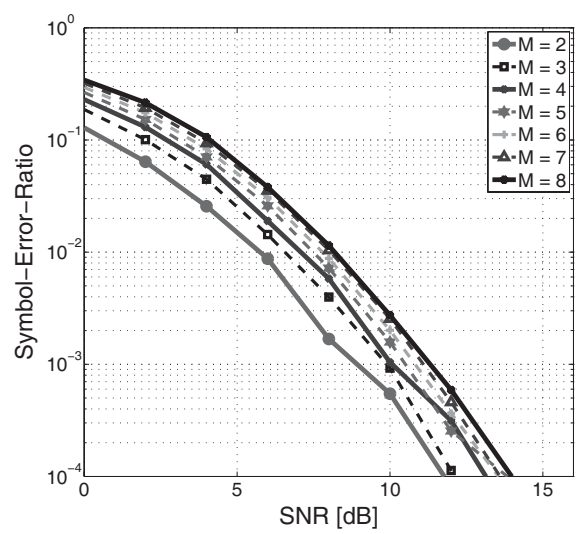

Fig. 2. SER of FBE-SM. Parametric study for different values of transmit antennas $M$. Setup: i) $S=4$, and ii) $n=4$.

to the error propagation effect discussed above. By comparing the best, $(S, n)=(4,4)$, and the worst, $(S, n)=(16,16)$, system setups shown in Fig. 1, we observe that the BER gets worse of approximately $1.5 \mathrm{~dB}$ at a BER of $10^{-3}$. However, this performance drop is compensated by a small increase, from $2.25 \mathrm{bits} / \mathrm{s} / \mathrm{Hz}$ to $2.3125 \mathrm{bits} / \mathrm{s} / \mathrm{Hz}$, in the achievable bit rate which is an increase of less than $3 \%^{1}$. This example demonstrates the effects of error propagation and shows $S=4$ and $\tilde{K}=2.25$ as a better choice than $S=16$ and $\tilde{K}=2.3125$ resulting from jointly optimising the steps in Section III-A.

In Figs. 2 and 3, we show the SER and BER of FBE-SM for various antennas at the transmitter, respectively. If $M=$ $2,4,8$, the system reduces to conventional SM. As expected [3], in Fig. 2 we notice that the SER gets monotonically worse for increasing values of $M$. However, this translates to an increase in the system bit rate from $1 \mathrm{bit} / \mathrm{s} / \mathrm{Hz}$ if $M=2$ to $3 \mathrm{bits} / \mathrm{s} / \mathrm{Hz}$ if $M=8$. When looking into Fig. 3, we observe that the BER does not get worse monotonically for increasing $M$. For example, the system setups with $M=5, M=6$, and $M=7$ offer a worse BER and a lower bit rate than the setup with $M=8$. This is mainly due to the error propagation effect of the FBE process. However, we also notice that for high SNRs, the performance difference between the setups with $M=5, M=6$ and the setup with $M=8$ is smaller. In particular, the $1.7 \mathrm{~dB}$ difference seen at a BER of $10^{-1}$ between $M=6$ and $M=8$, reduces to only $0.7 \mathrm{~dB}$ at a BER

\footnotetext{
${ }^{1}$ The bit rates are compared by considering only the number of transmit antennas since the modulation scheme in the signal domain is the same [1].
}

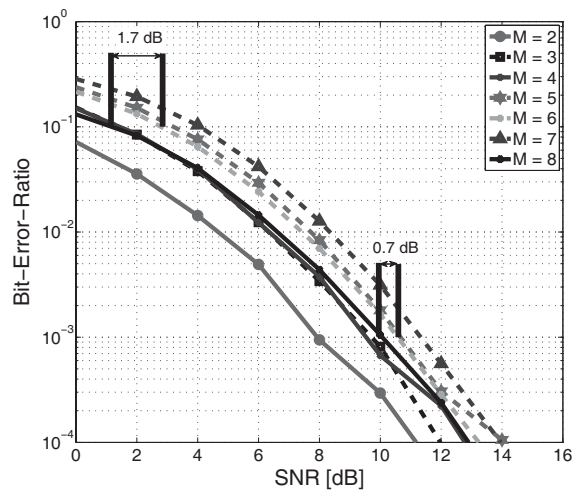

Fig. 3. BER of FBE-SM. Parametric study for different values of transmit antennas $M$. Setup: i) $S=4$, and ii) $n=4$.

of $10^{-3}$ as shown on Fig. 3. This is because error propagation is minimised for high SNRs. Even though the SM system with $M=8$ offers a better bit rate, this solution may not be practical due to cost and physical space constraints. This is a typical case where the proposed FBE-SM scheme with $M=5$ or $M=6$ can be an effective solution for trading-off bit rate for performance, e.g., with respect to the SM setup with $M=4$. In this context, it has to be mentioned that the results for a large $M$ are over-optimistic since no channel correlation is assumed. Naturally, if more antennas are fitted into a limited space, mutual coupling and correlation increases. In this Letter, we have not accounted for this effect as such investigation is beyond the scope of this work.

\section{CONCLUSions}

In this Letter, we have introduced a novel and more versatile SM scheme called FBE-SM. The method relies on the application of modulus conversion to achieve fractional bit rates, and allows any SM-MIMO wireless system to use an arbitrary number of antennas at the transmitter. Numerical results have also substantiated that the proposed method turns out to be a viable candidate to the design of compact mobile devices using $\mathrm{SM}$, by offering the desired degrees of freedom for trading-off performance, achievable bit rates, and cost.

\section{ACKNOWLEDGEMENT}

We greatfully acknowledge support from the Engineering and Physical Sciences Research Council (EP/G011788/1) for this work.

\section{REFERENCES}

[1] R. Y. Mesleh, H. Haas, S. Sinanovic, C. W. Ahn, and S. Yun, "Spatial modulation," IEEE Trans. Veh. Technol., vol. 57, no. 4, pp. 2228-2241, July 2008.

[2] J. Jeganathan, A. Ghrayeb, and L. Szczecinski, "Spatial modulation: optimal detection and performance analysis," IEEE Commun. Lett., vol. 12, no. 8, pp. 545-547, Aug. 2008.

[3] J. Jeganathan, A. Ghrayeb, L. Szczecinski, and A. Ceron, "Space shift keying modulation for MIMO channels," IEEE Trans. Wireless Commun., vol. 8, no. 7, pp. 3692-3703, July 2009.

[4] P. Wolniansky, G. Foschini, G. Golden, and R. Valenzuela, "V-BLAST: an architecture for realizing very high data rates over the rich-scattering wireless channel," in Proc. IEEE Int. Symp. Signals, Systems, and Electronics, pp. 295-300, Sep. 1998.

[5] W. L. Betts and K. D. Ko, "Fractional bit rate encoding in a pulse amplitude modulation communication system," U.S. Patent 6993067 , Jan. 31, 2006

[6] W. L. Betts, "Modulus converter for fractional rate encoding," U.S Patent 5103 227, Apr. 7, 1992.

[7] A. W. Paeth, Ed., Graphics Gems V. London: Academic Press, 1995. 